\title{
How Well Does AdS/QCD Describe QCD?
}

\author{
J. ERLICH \\ Physics Department, College of William and Mary \\ Williamsburg, VA, 23185, USA \\ E-mail: erlich@physics.wm.edu
}

\begin{abstract}
AdS/QCD is an extra-dimensional approach to modeling hadronic physics, motivated by the AdS/CFT correspondence in string theory. AdS/QCD models are often more accurate than would have been expected at energies below a few $\mathrm{GeV}$. We address the question of why these models are so successful, and respond to some of the criticisms that have been waged against these models.

Keywords: AdS/QCD, holographic QCD
\end{abstract}

\section{What is AdS/QCD?}

The AdS/CFT correspondence ${ }^{1}$ provides a powerful tool for extradimensional model-building. Qualitative features of electroweak symmetry breaking models with warped extra dimensions can often be predicted by analogy with the AdS/CFT correspondence. ${ }^{2}$ Even quantitatively, simple extra-dimensional models of QCD motivated by the AdS/CFT correspondence have proven successful at reproducing low-energy hadronic data like meson masses, decay constants, and coefficients of the chiral Lagrangian..$^{3-5}$ These models fall into two classes: top-down models based on brane constructions in string theory, and bottom-up models which are more phenomenological. Both top-down and bottom-up models in this framework are referred to as AdS/QCD models or holographic QCD.

AdS/QCD models are related to a number of earlier ideas which are useful for understanding properties of QCD at low energies: chiral symmetry breaking, hidden local symmetry, ${ }^{6}$ vector meson dominance, ${ }^{7}$ large $N,^{8}$ the Weinberg and Shifman-Vainshtein-Zakharov (SVZ) sum rules, ${ }^{9,10}$ Skyrmions, ${ }^{11}$ and matching of the low energy and high energy regimes. ${ }^{12}$ Aside from the pattern of chiral symmetry breaking, which is an input of some AdS/QCD models, aspects of all of the features listed above are a natural consequence of the extra-dimensional nature of these models. Hid- 
den local symmetry is related to the gauging of the global symmetry as suggested by the AdS/CFT correspondence; ${ }^{13}$ large $N$ is required by the classical limit of the AdS/CFT correspondence ${ }^{1}$ and is reflected in poles in correlation functions at real values of squared momenta; dominance of the lightest rho meson in hadronic couplings is due to oscillations of the heavier Kaluza-Klein modes in the extra dimensions; ${ }^{13,14}$ sum rules become exact relations for sums over Kaluza-Klein modes; ${ }^{4,5,15,16}$ solitonic solutions describing baryons in these models are closely related to the Skyrme model; ${ }^{17-19}$ resonance masses and decay constants conspire to reproduce the high energy behavior of correlators. ${ }^{20,21}$

The AdS/CFT correspondence relates certain strongly coupled large- $N$ gauge theories to weakly coupled theories containing gravity. ${ }^{1}$ The prototypical example is $\mathcal{N}=4$ supersymmetric $\mathrm{SU}(N)$ gauge theory in the limit $N \rightarrow \infty$ and 't Hooft coupling $g^{2} N \gg 1$, which is identified with Type IIB supergravity in an $\mathrm{AdS}_{5} \times \mathrm{S}^{5}$ spacetime background with nonvanishing 5 -form flux. The five-sphere $S^{5}$ plays an important role in matching supergravity fields to operators in the $\mathcal{N}=4$ gauge theory, but will not play an important role in our discussion of QCD-like models. Anti-de Sitter space in $4+1$ dimensions, $\mathrm{AdS}_{5}$, is described by the metric

$$
d s^{2}=\frac{R^{2}}{z^{2}}\left(\eta_{\mu \nu} d x^{\mu} d x^{\nu}-d z^{2}\right),
$$

where $\mu, \nu \in(0,1,2,3)$ and $\eta_{\mu \nu}$ is the Minkowski tensor in $3+1$ dimensions (4D). The coordinate $z$ is sometimes referred to as the radial coordinate. The region $z>0$ covers half (a Poincaré patch) of the full AdS spacetime, which is enough for our purposes. Although Anti-de Sitter space is a maximally symmetric spacetime, the radial coordinate plays a distinguished role in the metric Eq. (1). An interesting relationship between the radial coordinate and light-front dynamics has been observed by Brodsky and De Teramond (see, for example, Refs. ${ }^{22,23}$ ).

\subsection{The top-down approach}

In top-down AdS/QCD models, a brane construction in string theory is engineered which at low energies describes a gauge theory with features similar to QCD. ${ }^{17,24-27}$ The top-down model most similar to QCD is the Sakai-Sugimoto model, ${ }^{17,28}$ in which $N$ D4-branes in Type IIA string theory are wrapped on a circle, and are intersected by $N_{f}$ D8-branes and $N_{f}$ $\overline{\mathrm{D}}$-branes, as in Fig. 1. Open strings with both ends on the D4-branes describe a $\mathrm{SU}(N)$ gauge theory with sixteen supercharges. However, the 


$\underbrace{}_{\substack{\text { chiral } \\ \text { fermions }}}$ D4
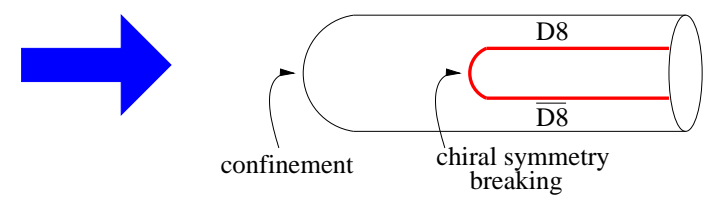

Fig. 1. The Sakai-Sugimoto model.

supersymmetry is broken if fermions are given antiperiodic boundary conditions around the circle, and at low energies the system is described by a pure $\mathrm{SU}(N)$ gauge theory with fermions and scalar fields whose masses are determined by the size of the circle. ${ }^{29}$ (Unfortunately, the mass gap is comparable to the masses of the extraneous fermions and scalars in this model.) The $\mathrm{D} 8$ and $\overline{\mathrm{D} 8}$-branes also break the supersymmetry. A string with one end on a D4-brane and one end on a D8-brane ( $\overline{\mathrm{D} 8}$-brane) describes a left-handed (right-handed) fermion in the effective 4D theory.

The separation of left and right-handed fermions makes chiral symmetry manifest in this theory, and makes the Sakai-Sugimoto model the AdS/CFT model of choice for describing QCD. The gravitational backreaction of the D4-branes generates a horizon, which is related to confinement as the horizon creates a mass gap in gravitational fluctuations which are interpreted as glueballs. ${ }^{29,30}$ Chiral symmetry breaking in the Sakai-Sugimoto model is due to the fact that the D8-branes and $\overline{\mathrm{D} 8}$-branes are joined together as a result of the curved spacetime geometry, as in Fig. 1. In this model the location at which the D8 and $\overline{\mathrm{D} 8}$-branes meet is an adjustable parameter and controls the ratio of confining to chiral symmetry breaking scales. ${ }^{31-33}$

\subsection{The bottom-up approach}

In bottom-up AdS/QCD models, we specify an extra-dimensional spacetime geometry and the fields that propagate in them based on the properties of QCD which we would like to be incorporated. Towers of KaluzaKlein modes are identified with towers of radial excitations of QCD states. Boundary conditions on gauge fields break the higher-dimensional gauge invariance, while the corresponding global symmetry remains in the effective $3+1$ dimensional theory. For example, an $\mathrm{SU}(2) \times \mathrm{SU}(2)$ gauge theory in $4+1$ dimensions (5D) can represent the approximate chiral symmetry of QCD. A set of scalar fields transforming in the fundamental representation of both $\mathrm{SU}(2)$ gauge groups can spontaneously break the symmetry to a diagonal subgroup which may be identified with isospin. This is the basic scenario in some basic bottom-up models. ${ }^{4,5}$ Rather than use scalar fields 
to break the chiral symmetry, modified spacetime geometry and boundary conditions can do the same. ${ }^{15,34} \mathrm{~A}$ more general approach includes the scalar fields with modified boundary conditions. ${ }^{35}$

For simplicity the spacetime geometry in bottom-up models is often chosen to be a slice of $\mathrm{AdS}_{5}$ with metric given by Eq. (1) between $z=\epsilon$ and $z=z_{m}$, where $\epsilon / z_{m} \ll 1$. Such models are called hard-wall models because of the sharp boundary at $z=z_{m} \cdot{ }^{4,5,36-38}$ The $\mathrm{AdS}_{5}$ spacetime has an $\mathrm{SO}(2,4)$ isometry which is broken only by the boundaries of the spacetime. The consequence is that the effective $3+1$ dimensional theory has an $\mathrm{SO}(2,4)$ symmetry at high energies which is identified with the conformal invariance of QCD at high energies. The conformal symmetry ensures that correlation functions of operators as derived in these models will have the form required by asymptotic freedom. However, other aspects of these models are not expected to be valid at energies above a few $\mathrm{GeV}$ where stringy effects become important in QCD, so the matching of certain predictions of AdS/QCD models to the UV is somewhat ad hoc.

In summary, a basic bottom-up model is described by an $\mathrm{SU}(2) \times \mathrm{SU}(2)$ gauge theory in a slice of $\mathrm{AdS}_{5}$ with bifundamental scalar field $X$ and action

$$
\begin{aligned}
S= & \int d^{4} x \int_{\epsilon}^{z_{m}} d z \sqrt{|g|}\left[-1 /\left(2 g_{5}^{2}\right) \operatorname{Tr}\left(L_{M N} L^{M N}+R_{M N} R^{M N}\right)\right. \\
& \left.+\operatorname{Tr}\left(\left|D_{M} X\right|^{2}-m_{X}^{2}|X|^{2}\right)\right]
\end{aligned}
$$

where Lorentz indices $M$ and $N$ run from 0 to 4 , and $L_{M N}$ and $R_{M N}$ are field stregths of the two sets of $\mathrm{SU}(2)$ gauge fields. The fields $X_{i j}, i, j \in$ $(1,2)$, have the quantum numbers of the scalar quark bilinear $\bar{q}_{L i} q_{R j}$, where the subscripts $L$ and $R$ refer to left and right-handed chirality, respectively, and $q_{1}$ and $q_{2}$ are the up and down quark fields, respectively. According to the AdS/CFT correspondence we would identify the mass $m_{X}$ of the field dual to an operator of scaling dimension $\Delta=3$, via ${ }^{39,40}$

$$
m_{X}^{2} R^{2}=\Delta(\Delta-4)=-3,
$$

where $R$ is the AdS curvature scale of Eq. (1). We may make this choice for definiteness, although the AdS/CFT correspondence is not strictly valid for this model and we would be ignoring renormalization effects which modify operator scaling dimensions away from the high energy regime.

The solutions to the classical equations of motion with vanishing gauge fields and $X$ field of the form $X_{0}(z)$ are

$$
X_{0}(z)=m_{q} z^{4-\Delta}+\frac{\sigma}{4 \Delta-8} z^{\Delta} .
$$


For $2<\Delta<4$, the term proportional to the parameter $m_{q}$ is nonnormalizable in the sense that the contribution to the action from the integral over $z$ diverges if the UV cutoff length $\epsilon \rightarrow 0$. On the other hand, the term proportional to $\sigma$ is normalizable. We will allow both normalizable and non-normalizable backgrounds for the field $X$. The factor of $1 /(4 \Delta-8)$ in Eq. (5) is motivated by the AdS/CFT identification of $\sigma$ as the (isospinpreserving) chiral condensate $\left\langle\bar{u}_{L} u_{R}\right\rangle=\left\langle\bar{d}_{L} d_{R}\right\rangle{ }^{41}$

The predictions of the model are insensitive to $\epsilon$ as long as $\epsilon / z_{m} \ll 1$. Hence, the free parameters in the model are $m_{X}, g_{5}, m_{q}, \sigma$, and $z_{I R}$. If we fix $m_{X}^{2}=-3 / R^{2}$ then there are four parameters in addition to the choice of boundary conditions on the gauge fields. We can also fix $g_{5}$ by extending AdS/QCD predictions to the UV and matching with perturbative QCD, leaving only three model parameters. ${ }^{4,5}$ However, this model is especially simplistic. For example, there could be a nontrivial potential for $X$, as in J. Kapusta's talk, ${ }^{42}$ and the geometry does not need to be Anti-de Sitter space. $^{34,43}$

Meson masses and decay constants are determined by the effective $3+1$ dimensional theory. The Kaluza-Klein modes of the gauge fields have the quantum numbers of vector and axial-vector mesons. The masses of the Kaluza-Klein modes are determined by the eigenvalues of the equations of motion together with the prescribed boundary conditions. The decay constants are determined by computing the mixing between the KaluzaKlein modes and the zero modes for the gauge fields. ${ }^{17,28}$ Alternatively, the AdS/CFT correspondence can be used to calculate the correlation function of products of currents, and the the decay constants are the residues of poles at the location of the Kaluza-Klein masses. ${ }^{4,5}$ Some of the early predictions of this model, with particular values of the parameters, appear in Table 1. Some of the coefficients of the chiral Lagrangian are related to these observables, and were calculated in the same model with a different choice of parameters in Ref. ${ }^{5}$

Numerous additional results in AdS/QCD models have been calculated. In a hard-wall AdS/QCD model including a strange quark mass parameter, predictions for masses and decay constants of light mesons containing strange quarks mostly agree with experimental central values at the few percent level. ${ }^{45}$ The $q^{2}$ dependence of form factors and moments of generalized parton distributions in various AdS/QCD models are in relatively good agreement with experiment up to a few $\mathrm{GeV}$ (see, for example, Refs. ${ }^{46-48}$ ). From the behavior of form factors at small $q^{2}$, these models predict that mesons have a larger size as determined by their charge distribution than 
Table 1. Predictions of a hard-wall model. For more details see Refs. ${ }^{4,44}$

\begin{tabular}{|c|c|c|}
\hline Observable & $\begin{array}{c}\text { Measured } \\
(\mathrm{MeV}, \text { central values })\end{array}$ & $\begin{array}{c}\text { Model } \\
(\mathrm{MeV})\end{array}$ \\
\hline$m_{\pi}$ & 139.6 & 141 \\
$m_{\rho}$ & 775.8 & 832 \\
$m_{a_{1}}$ & 1230 & 1220 \\
$f_{\pi}$ & 92.4 & 84.0 \\
$F_{\rho}^{1 / 2}$ & 345 & 353 \\
$F_{a_{1}}^{1 / 2}$ & 433 & 440 \\
$g_{\rho \pi \pi}$ & 6.03 & 5.29 \\
$m_{f_{2}}$ & 1275 & 1240 \\
\hline
\end{tabular}

by their momentum distribution. ${ }^{48}$

\section{How well does AdS/QCD work?}

The summary of our discussion thus far is that AdS/QCD models seem to be reasonably reliable for predicting observables at below a few $\mathrm{GeV}$, but tend to make poor predictions at higher energies. Until we better understand why certain models work especially well, we should at best trust only those predictions which are independent of model details such as the choice of spacetime geometry or boundary conditions.

According to the AdS/CFT correspondence, finite temperature physics can be studied by introducing a black hole into the higher-dimensional spacetime. From general properties of black hole horizons, a universal prediction of the ratio of shear viscosity $\eta$ to entropy density $s$ was discovered by Kovtun, Son and Starinets: ${ }^{49} \eta / s=1 /(4 \pi)$. Other universal relations have subsequently been found, such as a relation between electrical conductivity to charge susceptibility. ${ }^{50}$ The zero-temperature observables quoted earlier are not universal, but we can test their dependence on model parameters. In the hard-wall model defined in the previous section, allowing the effective dimension $\Delta$ of the operator $\bar{q} q$ to vary from its UV value $\Delta_{U V}=3$ is tantamount to varying the mass of the field $X$ via Eq. (4). The mass varies from the Breitenlohner-Freedman bound ${ }^{51} m_{X}^{2}=-4 / R^{2}$ for $\Delta=2$, to $m_{X}^{2}=0$ at $\Delta=4$. The dependence of some AdS/QCD predictions as a function of $\Delta$ over part of this range is shown in Fig. 2, holding fixed $m_{\pi}, f_{\pi}$ and $m_{\rho} \cdot{ }^{35}$ Note the insensitivity of hard-wall AdS/QCD predictions to the parameter $\Delta$. However, there is more sensitivity if the boundary conditions on the gauge fields in the IR are modified. ${ }^{35}$ If the geometry is allowed to vary in a certain class of AdS/QCD models, it seems that the AdS geometry provides an especially good fit to data, but certain predictions are relatively 

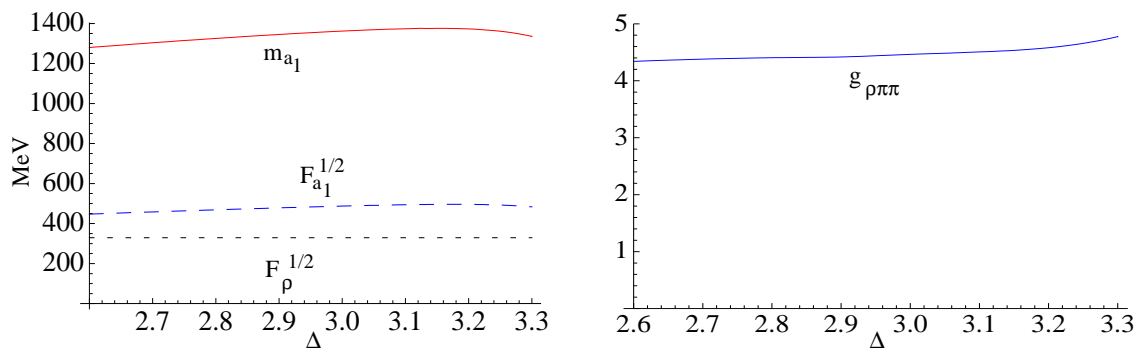

Fig. 2. AdS/QCD predictions are not sensitive to varying $\bar{q} q$ scaling dimension $\Delta$ around its UV value $\Delta_{U V}=3$, from Ref. ${ }^{35}$

insensitive to the details of the choice of geometry. ${ }^{43}$ The hard-wall model has a root-mean-squared error with seven observables and three parameters (as defined in Ref. ${ }^{4}$ ) of under 10\%. In general, AdS/QCD models are consistent with one another at around the 20-25\% level, and I am hopeful that we will better understand the unreasonable success of specific models.

\section{Challenges for AdS/QCD}

I would like to address some of the criticisms of the AdS/QCD approach that have been raised, both in the literature and in private (and not-soprivate) communication. I contend that most of these objections are red herrings if the scope of AdS/QCD models is properly understood, but AdS/QCD still faces some phenomenological challenges.

- $A d S / Q C D$ is not AdS/CFT.

The $\mathcal{N}=4$ Yang-Mills theory which serves as the prototypical example of the AdS/CFT correspondence is supersymmetric, conformal, and has a large number of colors and large 't Hooft coupling for calculability. This is all quite different from QCD, so why should we rely on the AdS/CFT correspondence to model QCD?

First of all, it is important to note that the existence of gravity duals is not tied to conformal invariance or supersymmetry. The Sakai-Sugimoto model is an example without either feature. The large- $N$ limit is required for a classical description with decoupled stringy physics, and the narrow resonances predicted by large $N$ are indeed features of classical AdS/QCD calculations. It would be interesting to pursue $1 / N$ corrections from quantum corrections in the 5D models, and as far as I know little work has been done in this regard (although there are close analogies to 5D models of electroweak symmetry breaking for which quantum corrections have been 
studied). For example, in AdS/QCD models the $\rho \pi \pi$ coupling has been calculated and compared with the decay rate of the rho meson. Such couplings can be used to self-consistently modify corresponding spectral functions.

The analogy with the AdS/CFT correspondence is useful for inferring qualitative features of AdS/QCD models, but these models stand on their own as 5D models independent of the AdS/CFT correspondence.

- The spectrum is not Regge-like.

Misha Shifman noted that hard-wall AdS/QCD models have spectra which depend on radial excitation number $n$ like $m_{n}^{2} \sim n^{2}$ as opposed to the Regge-like behavior $m_{n}^{2} \sim n .^{20}$ The soft-wall model ${ }^{52}$ obtains the correct Regge-like spectrum by introducing a dilaton background which modifies the equations of motion appropriately. At low energies the predictions of the soft-wall and hard-wall models are comparable.

- The model is matched to the UV, where QCD is weakly coupled.

In three places the hard-wall model described above is matched to the $\mathrm{UV}$ : in the choice of $\mathrm{AdS}_{5}$ geometry, in specifying the $5 \mathrm{D}$ gauge coupling $g_{5}$ by comparison with UV current correlators, and in assigning the squared mass $m_{X}^{2}=-3 / R^{2}$ to the scalar field by analogy with the AdS/CFT correspondence and the UV dimension of the operator $\bar{q} q$. Matching to the UV was done for definiteness, but one can allow parameters to vary, as in Ref. ${ }^{35}$ The conclusion is that matching to experiment prefers $g_{5}$ fixed to around the value obtained by matching to the UV, while there is less sensitivity to variation of $m_{X}$.

On a related note, Misha Shifman has pointed out that AdS/QCD predictions for correlators of isospin currents agree with Migdal's Padé approximation for the UV behavior of current correlators. ${ }^{20}$ The interesting thing about this approach is that one can systematically observe the radial direction of Anti-de Sitter space open up as the number of poles included in the Padé approximation increases. ${ }^{21}$

- Identification of QCD parameters depends on $N$.

The pattern of chiral symmetry breaking is built into the AdS/QCD models we have been discussing. If the AdS/CFT correspondence were strictly valid for this model, then $m_{q}$ and $\sigma$ of Eq. (5) would play the role of the source and expectation value of the operator $\bar{q}_{L} q_{R}$ dual to the scalar field $X$, up to an $N$-dependent rescaling. ${ }^{53}$ The $N$ dependence is not included in Eq. (5), so we should allow the possibility of rescaling $m_{q}$ and 
$\sigma$ appropriately. On the other hand, if we treat $m_{q}$ and $\sigma$ simply as model parameters, then the interpretation of those parameters as quark mass and chiral condensate is understood to be imprecise from the outset.

- AdS/QCD has incorrect high energy behavior.

Even though Anti-de Sitter space guarantees correct conformal behavior at high energies and the soft-wall model produces Regge-like spectra, there are many things AdS/QCD continues to get wrong at high energies. The density of states does not agree with the spectrum expected from string theory, and high-energy scattering amplitudes are not correctly predicted. ${ }^{54-56}$

We are forced at the moment to understand these models as low-energy descriptions valid only below some cutoff scale which may differ between models but is generally a few $\mathrm{GeV}$. It is still valuable to try to match certain aspects of these models to the UV, but at the same time we have to recognize the limitations of these models at high energies. Perhaps we can reconcile AdS/QCD predictions with high-energy scattering by including progressively more 5D fields into the model as the energy scale of interest increases, although so doing would introduce more model parameters and a decrease in predictivity.

- Important operators are not accounted for.

The glueball condensate is important for certain observables but is not included in some of these models. Simple AdS/QCD models like the hardwall and soft-wall models also do not include fields which would be dual to the operators $\bar{q}\left[\gamma^{i}, \gamma^{0}\right] T^{a} q$, which have significant overlap between vacuum and one-rho meson states. ${ }^{57}$ The basic response to such objections is that there is a natural way to include fields into the $5 \mathrm{D}$ theory which would describe corresponding operators and condensates in the effective 4D theory. The glueball condensate is related to the dilaton profile. New $5 \mathrm{D}$ fields can be included with quantum numbers conjugate to the operators in question, and one should study the effects of those fields. Any 5D field charged under the gauged isospin symmetry will lead to new couplings involving the rho meson which can be compared with experiment. At higher energies, more $5 \mathrm{D}$ fields are expected to be required in order to describe higher-dimension $4 \mathrm{D}$ operators that become important at those scales.

- AdS/QCD is an uncontrolled expansion.

If we understand AdS/QCD as a low-energy effective theory, then we should expect quantum corrections to generate higher-dimension operators 
in the $5 \mathrm{D}$ action suppressed by some scale. In AdS/QCD the relevant scale is the confining scale (or the chiral symmetry breaking scale, which is comparable). However, we are interested in physics above that scale. This is an honest difficulty for AdS/QCD models, and deserves further exploration.

Higher-dimension operators are generated by integrating out fields above the scale of interest. In the large- $N$ limit with large 't Hooft coupling, high-spin states decouple from the theory. ${ }^{\text {a }}$ Perhaps the effectiveness of the large- $N$ expansion in this limit is also related to the effectiveness of the AdS/QCD approach at low energies, summing only over "supergravity states" with spin less than or equal to two.

\section{Conclusions}

In summary, AdS/QCD models are extra-dimensional models of QCD resonances, and are generally accurate below a few $\mathrm{GeV}$ at the $10-25 \%$ level depending on the details of the model. The benefit of an extra-dimensional approach is that several features of QCD are immediate consequences of extra dimensions. QCD sum rules, vector meson dominance, and hidden local symmetry are all natural features of extra-dimensional models. A number of criticisms have been raised which challenge the AdS/QCD approach, based on the comparison of AdS/QCD models with the AdS/CFT correspondence in string theory and based on flawed phenomenology of these models, especially at high energies. Most of these objections disappear if we understand AdS/QCD as a class of effective low-energy models independent of the AdS/CFT correspondence.

It is an honor and a pleasure to have been part of this wonderful conference celebrating the vast achievements of Misha Shifman, who shows no sign of slowing down. Misha has played an important role in identifying challenges for AdS/QCD and has influenced the development of this field over the past few years. Thanks, and happy birthday Misha.

\section{References}

1. J. M. Maldacena, Adv. Theor. Math. Phys. 2, 231 (1998) [Int. J. Theor. Phys. 38, 1113 (1999)] [arXiv:hep-th/9711200].

2. N. Arkani-Hamed, M. Porrati and L. Randall, JHEP 0108, 017 (2001) [arXiv:hep-th/0012148].

3. G. F. de Teramond and S. J. Brodsky, Phys. Rev. Lett. 94, 201601 (2005) [arXiv:hep-th/0501022].

${ }^{\mathrm{a}}$ I thank Kaustubh Agashe for discussion on this point. 
4. J. Erlich, E. Katz, D. T. Son and M. A. Stephanov, Phys. Rev. Lett. 95, 261602 (2005) [arXiv:hep-ph/0501128].

5. L. Da Rold and A. Pomarol, Nucl. Phys. B 721, 79 (2005) [arXiv:hep$\mathrm{ph} / 0501218]$.

6. M. Bando, T. Kugo, S. Uehara, K. Yamawaki and T. Yanagida, Phys. Rev. Lett. 54, 1215 (1985)

7. J. J. Sakurai, Phys. Rev. Lett. 22, 981 (1969).

8. G. 't Hooft, Nucl. Phys. B 72, 461 (1974).

9. S. Weinberg, Phys. Rev. Lett. 18, 507 (1967).

10. M. A. Shifman, A. I. Vainshtein and V. I. Zakharov, Nucl. Phys. B 147, 385 (1979).

11. T. H. R. Skyrme, Nucl. Phys. 31, 556 (1962).

12. A. A. Migdal, Annals Phys. 109, 365 (1977).

13. D. T. Son and M. A. Stephanov, Phys. Rev. D 69, 065020 (2004) [arXiv:hep$\mathrm{ph} / 0304182]$.

14. S. Hong, S. Yoon and M. J. Strassler, arXiv:hep-ph/0501197.

15. J. Hirn and V. Sanz, JHEP 0512, 030 (2005) [arXiv:hep-ph/0507049].

16. J. Hirn and V. Sanz, Phys. Rev. D 76, 044022 (2007) [arXiv:hep-ph/0702005].

17. T. Sakai and S. Sugimoto, Prog. Theor. Phys. 113, 843 (2005) [arXiv:hepth/0412141].

18. K. Nawa, H. Suganuma and T. Kojo, Phys. Rev. D 75, 086003 (2007) [arXiv:hep-th/0612187].

19. A. Pomarol and A. Wulzer, arXiv:0904.2272 [hep-ph].

20. M. Shifman, arXiv:hep-ph/0507246.

21. J. Erlich, G. D. Kribs and I. Low, Phys. Rev. D 73, 096001 (2006) [arXiv:hepth/0602110].

22. S. J. Brodsky and G. F. de Teramond, Phys. Lett. B 582, 211 (2004) [arXiv:hep-th/0310227].

23. G. F. de Teramond and S. J. Brodsky, Phys. Rev. Lett. 102, 081601 (2009) [arXiv:0809.4899 [hep-ph]].

24. I. R. Klebanov and M. J. Strassler, JHEP 0008, 052 (2000) [arXiv:hepth/0007191].

25. M. Kruczenski, D. Mateos, R. C. Myers and D. J. Winters, JHEP 0405, 041 (2004) [arXiv:hep-th/0311270].

26. J. Babington, J. Erdmenger, N. J. Evans, Z. Guralnik and I. Kirsch, Phys. Rev. D 69, 066007 (2004) [arXiv:hep-th/0306018].

27. E. Antonyan, J. A. Harvey, S. Jensen and D. Kutasov, arXiv:hep-th/0604017.

28. T. Sakai and S. Sugimoto, Prog. Theor. Phys. 114, 1083 (2005) [arXiv:hepth/0507073].

29. E. Witten, Adv. Theor. Math. Phys. 2, 505 (1998) [arXiv:hep-th/9803131].

30. C. Csaki, H. Ooguri, Y. Oz and J. Terning, JHEP 9901, 017 (1999) [arXiv:hep-th/9806021].

31. E. Antonyan, J. A. Harvey and D. Kutasov, Nucl. Phys. B 784, 1 (2007) [arXiv:hep-th/0608177].

32. O. Aharony, J. Sonnenschein and S. Yankielowicz, Annals Phys. 322, 1420 (2007) [arXiv:hep-th/0604161]. 
33. C. D. Carone, J. Erlich and M. Sher, Phys. Rev. D 76, 015015 (2007) [arXiv:0704.3084 [hep-th]].

34. J. Hirn, N. Rius and V. Sanz, Phys. Rev. D 73, 085005 (2006) [arXiv:hep$\mathrm{ph} / 0512240]$.

35. J. Erlich and C. Westenberger, Phys. Rev. D 79, 066014 (2009) [arXiv:0812.5105 [hep-ph]].

36. J. Polchinski and M. J. Strassler, arXiv:hep-th/0003136.

37. A. Karch, E. Katz and N. Weiner, Phys. Rev. Lett. 90, 091601 (2003) [arXiv:hep-th/0211107].

38. S. Hong, S. Yoon and M. J. Strassler, JHEP 0604, 003 (2006) [arXiv:hepth/0409118].

39. E. Witten, Adv. Theor. Math. Phys. 2, 253 (1998) [arXiv:hep-th/9802150].

40. S. S. Gubser, I. R. Klebanov and A. M. Polyakov, Phys. Lett. B 428, 105 (1998) [arXiv:hep-th/9802109].

41. I. R. Klebanov and E. Witten, Nucl. Phys. B 556, 89 (1999) [arXiv:hepth/9905104].

42. T. Gherghetta, J. I. Kapusta and T. M. Kelley, Phys. Rev. D 79, 076003 (2009) [arXiv:0902.1998 [hep-ph]].

43. D. Becciolini, M. Redi and A. Wulzer, arXiv:0906.4562 [hep-ph].

44. E. Katz, A. Lewandowski and M. D. Schwartz, Phys. Rev. D 74, 086004 (2006) [arXiv:hep-ph/0510388].

45. E. Katz, plenary talk at Lattice 2008 conference. Talk posted at http://conferences.jlab.org/lattice2008/plenary.html

46. H. J. Kwee and R. F. Lebed, Phys. Rev. D 77, 115007 (2008) [arXiv:0712.1811 [hep-ph]].

47. H. R. Grigoryan and A. V. Radyushkin, Phys. Lett. B 650, 421 (2007) [arXiv:hep-ph/0703069].

48. Z. Abidin and C. E. Carlson, Phys. Rev. D 77, 095007 (2008) [arXiv:0801.3839 [hep-ph]].

49. P. Kovtun, D. T. Son and A. O. Starinets, Phys. Rev. Lett. 94, 111601 (2005) [arXiv:hep-th/0405231].

50. P. Kovtun and A. Ritz, Phys. Rev. D 78, 066009 (2008) [arXiv:0806.0110 [hep-th]].

51. P. Breitenlohner and D. Z. Freedman, Annals Phys. 144, 249 (1982).

52. A. Karch, E. Katz, D. T. Son and M. A. Stephanov, Phys. Rev. D 74, 015005 (2006) [arXiv:hep-ph/0602229].

53. A. Cherman, T. D. Cohen and E. S. Werbos, arXiv:0804.1096 [hep-ph].

54. D. M. Hofman and J. Maldacena, JHEP 0805, 012 (2008) [arXiv:0803.1467 [hep-th]].

55. C. Csaki, M. Reece and J. Terning, JHEP 0905, 067 (2009) [arXiv:0811.3001 [hep-ph]]

56. B. Pire, C. Roiesnel, L. Szymanowski and S. Wallon, Phys. Lett. B 670, 84 (2008) [arXiv:0805.4346 [hep-ph]].

57. L. Y. Glozman, arXiv:0903.3923 [hep-ph]. 